\section{Acrylamide Capture of DNA-Bound Complexes: Electrophoretic Purifica- tion of Transcription Factors}

BioTechniques 32:808-815 (April 2002)

\begin{abstract}
We have developed a rapid nonradioactive electrophoretic technique to analyze proteins within DNA-binding complexes, acrylamide capture of DNA-binding complexes (ACDC), using Acrydite ${ }^{T M}$-linked DNA-binding targets. The method is highly sensitive and easily adaptable to virtually any proteinDNA interaction. The utility of this technique is illustrated using recombinant and fulllength androgen receptors and associated co-regulatory proteins present within nuclear extracts. In brief, proteins were incubated with DNA-binding targets in which one oligonucleotide was synthesized with an Acrydite moiety at the $5^{\prime}$ end to allow for covalent linkage to acrylamide. Alternatively, gene promoter regions were amplified with an Acrydite-modified PCR primer to analyze protein-DNA complexes. The DNA-binding reaction was polymerized into an acrylamide matrix within the well of a precast gel. Proteins complexed to the Acrydite DNA are trapped and purified by the electrophoretic migration of unbound proteins. Proteins captured in the Acrydite-DNA can be eluted and identified by Western analysis or 2-D gel electrophoresis. The advantages of this technique are that it is rapid, adaptable, sensitive, unlimited by the size of the DNA or protein complex, and can be used to detect tertiary interactions with co-regulatory factors and unidentified proteins. These features make the ACDC technique a powerful tool for transcription factor research.
\end{abstract}

\section{INTRODUCTION}

To investigate transcriptional regulation, it is essential to identify and characterize transcription factors and their co-activators and co-repressors that bind to specific regulatory DNA sequences within a promoter region. The most widely applied methods to investigate DNA-protein interactions include the electrophoretic mobility shift assay (EMSA) and solution-based DNA affinity chromatography (3-5). In the EMSA method, a fragment of DNA is radiolabeled, purified, and then incubated with either a recombinant protein(s) of interest or a nuclear extract. After the binding reaction has reached equilibrium, it is loaded onto a non-denaturing gel and electrophoresed under low ionic strength. The gel is dried and exposed to autoradiographic film. The unbound radiolabeled DNA migrates in the electric field at a faster rate than the DNA probe that is bound to the protein(s) of interest. The protein-bound DNA migrates at a rate primarily influenced by the mass and characteristics of the bound protein(s) and, to a lesser degree, by the length of the DNA probe. Of great importance to the success of the EMSA technique is that the DNAbound protein does not have an appreciable dissociation from the DNA once the complex enters the polyacrylamide. Dissociation is inhibited because of the commonly known "cage-effect" that functions to stabilize the interaction compared with the dissociation in solution (10). However, the window of resolution between the free DNA and bound complexes to be studied is limited by the length of the DNA $(<300 \mathrm{bp})$ and the size of the protein(s) $(<400$ $\mathrm{kDa}$ ). Larger protein-DNA complexes are difficult to resolve or do not effectively enter the gel. EMSA is not well suited for analyzing tertiary co-regulator complexes that dissociate from the DNA-bound transcription factors during electrophoresis, presumably because these protein-protein interactions are not stable enough to withstand migration through a polyacrylamide gel.

Two techniques have been described to identify the protein bound to DNA in an EMSA. First, an antibody can be included in the binding reaction to cause a supershift in the protein-bound DNA in EMSA gels. Unfortunately, this technique is not very reliable for most antibodies. Second, the EMSA can be transferred to a membrane and probed with an antibody to determine if the bound DNA band corresponds to the protein of interest (1). This approach is limited in sensitivity and requires the overexpression of the protein of interest within the extract.

DNA affinity chromatography can be employed to isolate proteins that bind specifically to DNA for further identification and characterization. Concatermerized target DNA is covalently linked to activated cyanogen bromide sepharose (5). Protein extracts are incubated with the immobilized DNA and washed to remove nonspecific binding proteins, followed by the elution of the specifically bound proteins. This method requires a relatively large amount of starting material and is labor intensive. In addition, a considerable amount of the specific protein of interest is lost during the wash steps because of the dissociation of the desired complex in solution, which is faster compared to the "caged interaction" in an EMSA. Because of the time, effort, and expense, this method would be impractical if numerous interactions were to be studied simultaneously.

Here we describe a new method, acrylamide capture of DNA-binding complexes (ACDC), to isolate and identify sequence-specific DNA-binding proteins from relatively small samples. This method combines in part the advantages of EMSA and DNA affinity chromatography but is more rapid and effective at identifying DNA-bound proteins and less constrained by the size and abundance of DNA and protein complexes. The method employs a new technological development in which oligonucleotides are modified during synthesis to have an Acrydite ${ }^{\mathrm{TM}}$ moiety (Mosaic Technologies, Waltham, MA, USA) on the $5^{\prime}$ end that allows for the covalent linkage of the oligonucleotide to acrylamide upon polymerization. The Acrydite oligonucleotide modification was developed to trap RNA molecules in a sequence-specific manner by polymerizing an "antisense Acrydite oligonucleotide" into an acrylamide layer within a polyacrylamide gel (6). We have expanded the Acrydite-based technology to trap the DNA-binding proteins by using annealed dsDNA molecules in which one oligonucleotide strand contains an Acrydite group. In this way, we can immobilize dsDNA within a polyacrylamide matrix. The protein complexes bound to the DNA can then be purified by the elec- 
trophoretic removal of the unbound proteins. The retained proteins can be rapidly eluted and probed by Western analysis for known proteins of interest or by 2-D gel electrophoresis and mass spectrometry for unidentified proteins. The technique has also proven useful for detecting co-regulator proteins that form tertiary complexes with transcription factors bound to the DNA.

\section{MATERIALS AND METHODS}

A 5\% polyacrylamide (37.5:1; polyacrylamide: bisacrylamide) $0.5 \times \mathrm{TBE}$ (89 mM Tris, pH 8.3, 45 mM boric acid, 2 mM EDTA) gel was cast using a miniprotean II $^{\mathrm{TM}}$ apparatus (Bio-Rad Laboratories, Hercules, CA, USA). Oligonucleotides containing the Acrydite moiety (Ac) corresponding to the NF-1 consensus sequence (5'-AcTGTGAGTTTTGGATTGAAGCCAATATGATAGGA-3') (NF1-Ac), a high-affinity androgen response element (ARE) (5'-AcGAGTGTGAGGGTACACGGTGTTCTTGTGGATGT-3') (ARE-Ac), and the respective complementary oligonucleotides without an Acrydite modification were supplied by Mosaic Technologies. The oligonucleotides were annealed by heating at $95^{\circ} \mathrm{C}$ for $2 \mathrm{~min}$ and then slowly cooled to room temperature. The annealed Acrydite target DNA sequence was efficiently linked to the polyacrylamide gel during polymerization, as assessed by ethidium bromide staining (data not shown). Recombinant protein-DNA-binding reactions were prepared as follows. Six micrograms of His-Tag rat androgen receptor DNA-binding domain (AR-DBD) (3 $\mu \mathrm{L}$; amino acids 524-648) were incubated in $15 \mu \mathrm{L}$ buffer D (20 mM HEP$\mathrm{ES}, \mathrm{pH} 7.8,100 \mathrm{mM} \mathrm{KCl}, 10 \%$ glycerol, $1 \mathrm{mM}$ DTT) with $2 \mu \mathrm{g}$ poly dI-dC and $3.5 \mu \mathrm{L} 30 \%$ acrylamide:bisacrylamide (37.5:1) in the presence of $1 \mu \mathrm{L}$ buffer D, $1 \mu \mathrm{L}$ NF1-Ac (final concentration, $1 \mu \mathrm{M}$ ), or $1 \mu \mathrm{L}$ ARE-Ac (final concentration, $1 \mu \mathrm{M})$. Samples were incubated for $15 \mathrm{~min}$ at room temperature, and then $1.25 \mu \mathrm{L}$ of $5 \%$ ammonium persulfate (APS) and TEMED mixture (diluted 1:30 in buffer D) were added, mixed briefly, and loaded into a well of the previously cast $5 \%$ polyacrylamide gel. Polymerization was complete with- in 5 min. Running buffer $(0.5 \times$ TBE $)$ was added, wells were washed thoroughly, and electrophoresis was carried out at a $15 \mathrm{~V} / \mathrm{cm}$ constant voltage for 1 h. The polyacrylamide fragments containing the covalently linked Acrydite DNA/protein complexes were excised, transferred to $70 \mu \mathrm{L}$ sample buffer $(62.5$ $\mathrm{mM}$ Tris- $\mathrm{HCl}, \mathrm{pH} 6.8,2 \%$ SDS, $10 \%$ glycerol, $0.2 \mathrm{mg} / \mathrm{mL}$ bromophenol blue,
$5 \% \beta$-mercaptoethanol), and incubated at $90^{\circ} \mathrm{C}$ for $4 \mathrm{~min}$. A $50-\mu \mathrm{L}$ aliquot of the samples was run on a $12.5 \%$ SDSpolyacrylamide gel, transblotted to a PVDF membrane (Millipore, Bedford, MA, USA), and probed for His-Tag rat AR-DBD using the penta-His antibody (Qiagen, Valencia, CA, USA).

To examine the utility of this method in a more complex analysis, we 
used forward and reverse universal primers with one containing a $5^{\prime} \mathrm{Ac}$ (FUP-Ac, 5'-AcGTTTTCCCAGTCACGAC- $3^{\prime}$ and RUP-Ac, 5'-AcCAGGAAACAGCTATGAC-3') to PCR-amplify from the -286 to +28 region of the rat Probasin promoter (PB) cloned into the HindIII and BamHI sites of pBluescript $^{\circledR}$ (Stratagene, La Jolla, CA, USA). In addition, the multiple cloning site of pBluescript was amplified with these primers for use as a nonspecific DNA control. The PCR products were purified by a spin column (Qiagen). Binding reactions were carried out as described earlier, except that we used nuclear extracts from $\mathrm{LNCaP}$ cells prepared as previously described (2). The specific protein complex bound to the Acrydite DNA was eluted as described earlier and separated on an $8 \%$ SDSpolyacrylamide gel and immunoblotted for $\beta$-catenin (B-Cat), a known co-activator of AR using anti- $\beta$-catenin antibody (Santa Cruz Biotechnology, Santa Cruz, CA, USA) (9).
Alternatively, proteins were eluted from the ACDC assay and analyzed using 2-D gel electrophoresis, followed by silver staining with the following modifications. For these experiments, binding reactions were scaled up in volume to include $200 \mu \mathrm{g} \mathrm{LNCaP}$ nuclear extract in a final volume of $160 \mu \mathrm{L}$. The samples were polymerized into the wells of a $13.5 \times 20 \mathrm{~cm}$ precast $8 \%$ polyacrylamide gel (1.5 $\mathrm{mM}$ spacers) and run at $15 \mathrm{~V} / \mathrm{cm}$ for $3 \mathrm{~h}$. Proteins were eluted from the excised fragments in $250 \mu \mathrm{L}$ isoelectric focusing buffer minus urea (4\% CHAPS, $100 \mathrm{mM}$ DTT) at $70^{\circ} \mathrm{C}$. Urea was then added to a final concentration of $8 \mathrm{M}$. Each sample (200 $\mu \mathrm{L}$ ) plus $0.1 \%$ biolytes (Bio-Rad Laboratories) was used to hydrate overnight first-dimension isoelectric focusing gel strips with $\mathrm{pH}$ gradients from $\mathrm{pH}$ 3.0-10.0 (Bio-Rad Laboratories). The first dimension was focused for 15000 Volt hours. The second dimension was resolved on 12\% SDS-PAGE and silverstained as described previously (8).

\section{RESULTS}

In our newly developed ACDC method, we have incubated the DNAbinding site targets containing an Acrydite modification with a protein(s) of interest in a standard DNA-binding buffer containing acrylamide. After the reaction reached equilibrium, APS and TEMED were added to immobilize the DNA and bound protein complexes. The reaction was immediately transferred into the well of a precast non-denaturing polyacrylamide gel where it completed polymerization. We added an electrophoresis buffer and applied an electric current. Proteins that were not specifically bound to the double-stranded Acrydite oligonucleotide were electrophoresed away from the specifically bound complex. This electrophoretic purification resulted in a sequence-specific retention of DNA-binding proteins that were probed directly by Western analysis for known protein(s). This method could potentially be adapted for

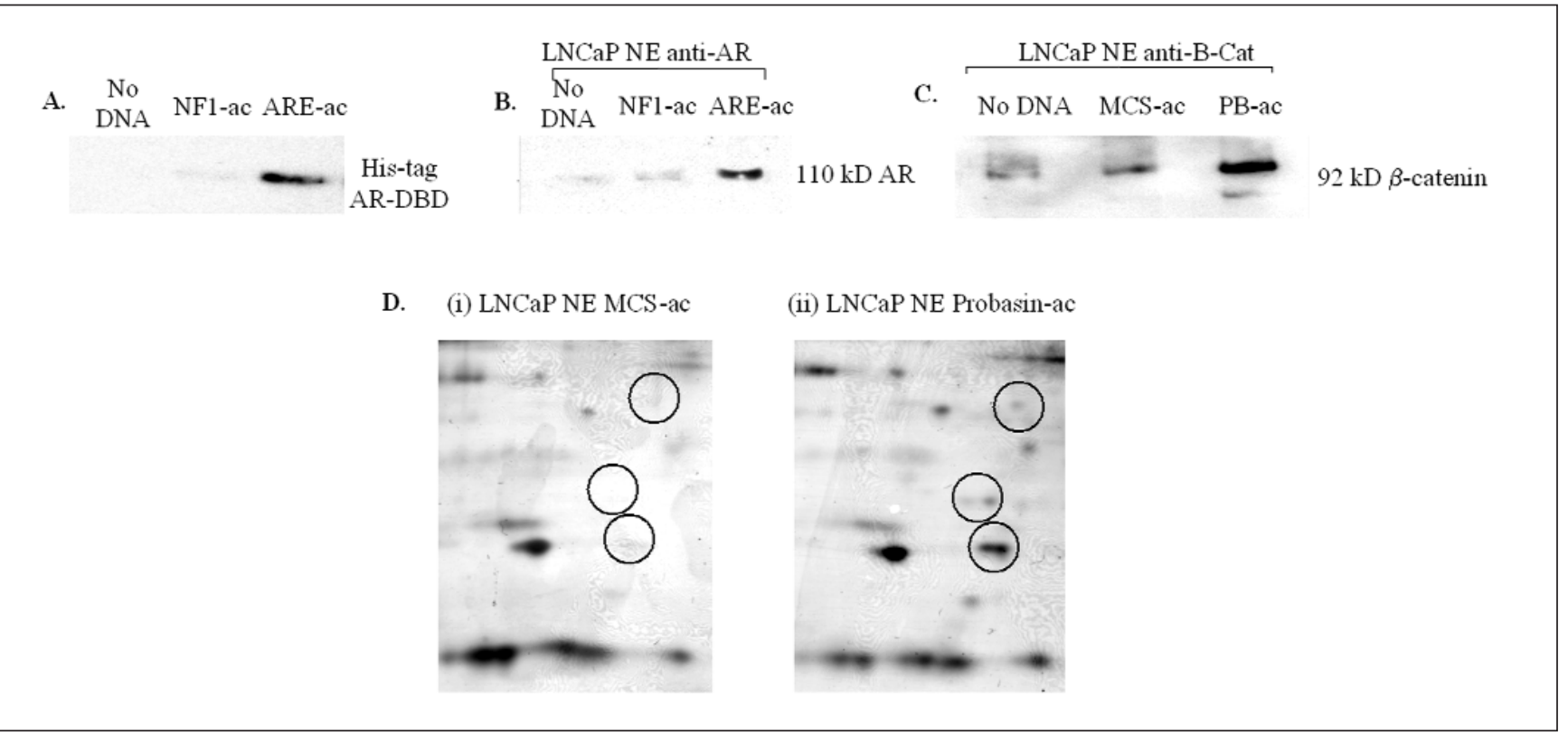

Figure 1. Specific retention of proteins to AR promoter regions using the ACDC method. (A) The ACDC assay was carried out by incubating recombinant His-tag AR-DBD without DNA or with an NF1-Ac binding site or ARE-Ac. The binding reaction was polymerized in the well of a precast polyacrylamide gel and electrophoresed. The protein was extracted from the Acrydite gel, run on a 12.5\% SDS-polyacrylamide gel, and immunoblotted with anti-His tag antibody. Retention of the His tag AR-DBD was observed only for the specific ARE-Ac binding site and not for the non-cognate NF1-Ac binding site or in the absence of DNA. (B) The ACDC method was used to capture the full-length AR. For this experiment, LNCaP nuclear extracts (NEs) treated with the synthetic androgen R1881 were incubated without DNA, NF1-Ac, or ARE-Ac. After electrophoresis to separate out the unbound protein, the specifically bound protein was extracted from the Acrydite gel, separated on an $8 \%$ SDS-polyacrylamide gel, and immunoblotted with an anti-AR-DBD antibody. (C) The ACDC assay was used to capture B-Cat from LNCaP nuclear extract treated with R1881. Nuclear extract was incubated without DNA, with MCS-Ac, or with PB-Ac (-286 to +28 ) that were created by PCR amplification using specific primers with a $5^{\prime} \mathrm{Ac}$ attached. Retention of the 92-kDa B-Cat for the AR-containing LNCaP nuclear extract was observed only for PB-Ac, which contains four known AREs. (D) LNCaP NEs were incubated with (i) MCS-Ac or (ii) PB-Ac. Binding reactions were polymerized and bound proteins were electrophoretically purified. Proteins were eluted, subjected to 2-D gel electrophoresis, and silver-stained. Proteins from the LNCaP nuclear extract specifically retained in the binding reaction containing the PB-Ac are illustrated. 
$\mathrm{N}$-terminal sequencing or mass spectroscopy analysis for unknown proteins isolated by 2-D electrophoresis.

To illustrate the specificity of the method, we have incubated a recombinant His-tagged AR-DBD with an ARE-Ac, NF1-Ac, or without DNA. The binding reactions were polymerized and electrophoresed as described earlier. Protein retained in the Acrydite layer was eluted and separated on an SDSpolyacrylamide gel, followed by Western analysis with an anti-His tag antibody. These data demonstrate the sequence-specific retention of the recombinant AR-DBD to the ARE-Ac oligonucleotides (Figure 1A). The specificity of retention was optimal below $0.3 \mu \mathrm{g}$ total protein input per microliter of reaction volume. Increases beyond this concentration resulted in protein precipitation and nonspecific retention of the protein in the Acrydite layer regardless of the presence of DNA.
Next, we applied the ACDC technique to a complex protein mixture to determine if we could specifically retain the full-length AR from a nuclear extract of the prostate cancer cell line LNCaP, which expresses AR at a relatively low level. Western analysis of the electrophoretically purified complex demonstrated a specific retention of the endogenous full-length AR from this complex mixture (Figure 1B). The AR, like most transcription factors, is known to bind to several co-regulatory proteins that bridge with the basic transcriptional machinery to facilitate transcription. The interactions of co-regulators are most often studied in yeast two-hybrid and affinity pull-down systems that do not include the native transcription factor DNA-binding site. As mentioned earlier, EMSA is largely ineffective in detecting co-regulators, presumably because the protein-protein interactions between co-regulators and transcription factors are destabilized by migration through the polyacrylamide matrix. With the ACDC method, the experimenter is no longer constrained by the length of DNA, the size of the complex formed, or the number of interactions on a fragment of DNA because the complexes of interest do not need to be resolved by the electrophoretic migration of the complex. Therefore, this technique theoretically can be applied to large promoter fragments to analyze the host of protein factors that bind in a sequence-specific manner and the tertiary interactions of co-regulators.

To create a versatile approach to investigate interactions on a promoter, we have used a FUP-Ac and RUP that flank multiple cloning sites (MCSs) within many common plasmid vectors. In this technique, FUP-Ac was used in combination with an unmodified RUP in a reaction to amplify a promoter of interest. The PCR amplification of the 
MCS of pBluescript with FUP-Ac and RUP was used as a control DNA. We have applied this technique to study the DNA-protein interactions of the AR on the rat $\mathrm{PB}$ promoter. To determine if the ACDC method captured co-regulator protein interactions, we incubated LNCaP NEs with the PCR-amplified PB-Ac (from -286 to +28 ) that contains four known AREs (7), the pBluescript multiple cloning site (MCS-Ac), or in the absence of DNA. A Western analysis for the B-Cat, which is known to interact with the AR (9), demonstrated the specific retention of this co-regulator in the reaction containing the $\mathrm{PB}$ Ac (Figure 1C). Therefore, the ACDC method allows for the rapid analysis of not only protein-DNA interactions but also tertiary interactions with co-regulators that are specifically captured in the Acrydite layer. Since the tertiary interactions of DNA-bound proteins with co-regulators are very difficult to detect in conventional EMSA, the ACDC provides a new approach to investigate specific co-regulator interactions as they occur on the DNA in a rapid and efficient manner.

To identify unknown proteins that may interact with a promoter of interest or with a transcription factor bound to a specific DNA sequence, the ACDC can be adapted to further characterization by 2-D gel electrophoresis and/or mass spectrometry. To illustrate proteins captured by specific DNA sequences, we have analyzed proteins from $\mathrm{LNCaP}$ nuclear extracts with the ACDC technique using 2-D gel electrophoresis. The ARE-Ac and PB-Ac were used to capture interacting proteins in comparison with a reaction that did not contain DNA. Following the ACDC purification, proteins were eluted and analyzed by 2-D electrophoresis, followed by silver staining (Figure 1D). These results demonstrate that the ACDC method can reveal proteins uniquely captured on specific DNA elements in quantities that could be identified by mass spectrometry (8). It is also evident that particular proteins at this concentration within the reaction volume are retained in the Acrydite layer regardless of DNA interactions, presumably because of either lack of sufficient charge to migrate out of the Acrydite layer or protein precipitation in the reaction. Background can be significantly reduced through empirical optimization of protein concentration and buffer conditions to enhance the potential power of this technique.

\section{DISCUSSION}

The ACDC technique provides a novel approach to investigate the interactions between DNA-binding proteins and specific DNA sequences. The technique is simple, rapid, and can be easily adapted to any protein-DNA interaction. In contrast with EMSA, the ACDC method is not restrained by the length of the DNA to be investigated or the size of the protein(s) or complexes of interest. In our preliminary work, we have shown that this technique can isolate recombinant DNA-binding proteins or transcription factors from complex mixtures such as a nuclear extract. A major advantage of the ACDC method over EMSA is that it allows for the identification of co-regulators bound to DNA-binding proteins, whereas these interactions are often unstable under conventional EMSA conditions. By using ACDC, the multimeric protein-DNA complex is trapped in polyacrylamide, and the tertiary interactions of co-regulator proteins with transcription factors are retained. This allows for the investigation of the potential differential usage of co-regulators on different promoters and the identification of proteins within the assembled enhanceosomes.

To optimize the utility of this technique, care should be taken in preparing recombinant proteins and nuclear extracts to avoid or remove insoluble protein precipitates because these can significantly increase the background of nonspecific retention. This is likely due to the ineffective electrophoretic migration of these precipitates caused by aggregation. In addition, as with EMSA, care must be taken not to overload the reaction with protein because the nonspecific retention of protein in the Acrydite layer may result. The ACDC assay is sensitive to this issue because the liquid-phase volume of the Acrydite-binding reaction decreases upon the polymerization of the binding reaction and may cause proteins to precipitate at high concentrations. Furthermore, large proteins of neutral charge may also in- crease nonspecific background. Dependent on the nature of the proteins of interest, the addition of low concentrations of ionic detergents such as SDS may aid in the migration of neutralcharge proteins without significant disruption to the protein-DNA complex.

The use of an Acrydite moiety attached to PCR primers (such as universal primers) should permit the rapid screening of large promoter sequences for specific transcription factor binding sites and the rapid discovery of DNAbinding proteins and transcriptional coregulators using the large repertoire of antibodies available. Furthermore, using an Acrydite universal primer and a sequence-specific primer within the promoter of interest, the rapid mapping of transcription factor binding sites can be achieved.

In summary, this novel technique allows for a simple and rapid approach to investigate DNA-protein interactions and to purify DNA-binding proteins that specifically bind to target DNA sequences for further analysis including Western analysis and peptide sequencing. In addition to the speed, ease, and efficiency of this technique, it is able to both detect tertiary interaction with proteins associated with transcription factors bound to DNA and direct protein-DNA interactions.

\section{REFERENCES}

1.Demczuk, S., M. Harbers, and B. Vennstrom. 1993. Identification and analysis of all components of a gel retardation assay by combination with immunoblotting. Proc. Natl. Acad. Sci. USA 90:2574-2578.

2.Dignam, J., R. Lebovitz, and R. Roeder. 1983. Accurate transcription initiation by RNA polymerase II in a soluble extract from isolated mammalian nuclei. Nucleic Acids Res. 11:1475-1489.

3.Fried, M. and D. Crothers. 1981. Equilibria and kinetics of the lac repressor-operator interactions by polyacrylamide gel electrophoresis. Nucleic Acids Res. 9:6505-6525.

4.Garner, M. and A. Revzin. 1981. A gel electrophoresis method for quantifying the binding of proteins to specific DNA regions: application to components of the Escherichia coli lactose operon regulatory system. Nucleic Acids Res. 9:3047-3060.

5.Kadonaga, J. and R. Tjian. 1986. Affinity purification of sequence-specific DNA binding proteins. Proc. Natl. Acad. Sci. USA 83:5889-5893.

6.Kenney, M., S. Ray, and T. Boles. 1998. Mutation typing using electrophoresis and gel im- 
mobilized Acrydite probes. BioTechniques 25:516-521.

7.Reid, K.J., S.C. Hendy, J. Saito, P. Sorenson, and C.C. Nelson. 2001. Two classes of androgen receptor elements mediate cooperativity through allosteric interactions. J. Biol. Chem. 276:2943-2952.

8.Shevchenko, A., M. Wilm, O. Vorm, and M. Mann. 1996. Mass spectrometric sequencing of proteins from silver-stained polyacrylamide gels. Anal. Chem. 68:850-858.

9.Trucia, C., S. Byers, and E. Gelmann. 2000. $\beta$-catenin affects androgen receptor transcriptional activity and ligand specificity. Cancer Res. 60:4709-4713.

10.Vossen, K. and M. Fried. 1997. Sequestration stabilizes lac repressor-DNA complexes during electrophoresis. Anal. Biochem. 245:85-92.

We gratefully acknowledge the generous provision of Acrydite oligonucleotides by Mosaic Technologies. Address correspondence to Dr. Colleen Nelson, The Prostate Centre at Vancouver General Hospital, 2660 Oak Street, Jack Bell Research Centre, Vancouver, BC V6H 3Z6, Canada. e-mail: ccnelson@interchange.ubc.ca

Received 18 June 2001; accepted 17 December 2001.

\section{Colleen Nelson, Stephen Hendy, Kim Reid, and John Cavanagh Vancouver General Hospital, Vancouver, BC, Canada}

\section{Quantitative Analysis of Phenol Oxidase Activity in Insect Hemolymph}

BioTechniques 32:815-823 (April 2002)

\footnotetext{
ABSTRACT

We describe a simple, inexpensive, and robust protocol for the quantification of phenol oxidase activity in insect hemolymph. Discrete volumes of hemolymph from Drosophila melanogaster larvae are applied to pieces of filter paper soaked in an L-3, 4-dihydroxyphenylalanine ( $L$ DOPA) solution. Phenol oxidase present in
}

the samples catalyzes melanin synthesis from the L-DOPA precursor, resulting in the appearance of a roughly circular melanized spot on the filter paper. The filter paper is then scanned and analyzed with image-processing software. Each pixel in an image is assigned a grayscale value. The mean of the grayscale values for a circular region of pixels at the center of the image of each spot is used to compute a melanization index (MI) value; the computation is based on a comparison to an external standard (India ink). Numerical MI values for control and experimental larvae can then be pooled and subjected to statistical analysis. This protocol was used to evaluate phenol oxidase activity in larvae of different backgrounds: wild-type, lozenge, hopscotch Tumorous-lethal (which induces the formation of large melanotic tumors), and body-color mutations ebony and yellow. Our results demonstrate that this assay is sensitive enough for use in genetic screens with $\mathrm{D}$. melanogaster and could conceivably be used for evaluation of MI from hemolymph of other insects.

\section{INTRODUCTION}

The phenol oxidase enzyme catalyzes the synthesis of melanin from any of several precursor molecules, including L-3, 4-dihydroxyphenylalanine (LDOPA) $(1,17)$. It has been demonstrated that the blood (hemolymph) of many insects, including the model system insect Drosophila melanogaster, contains phenol oxidase, either carried within specialized blood cells (hemocytes) and/or dissolved in solution (8). In $D$. melanogaster larvae, these specialized hemocytes are called crystal cells. In insects, melanin synthesis has been linked to several key biological processes, including sclerotization and darkening of cuticle and wings $(15,16)$. Melanization has also been implicated in humoral recognition of foreign pathogens, wound healing $(1,3,17)$, and melanotic encapsulation of, and cytotoxic effects on, self and nonself tissue (9). However, the exact role of melanin in these processes and the genetic and regulatory mechanisms that govern its synthesis are only partially understood. It should be possible to identify the genes involved in the regulation of melanin synthesis, transport, and deposition by screening large numbers of novel genetic back- 